\title{
Investigación transmedia. Cultura participativa en la creación del conocimiento académico
}

\section{Transmedia research. Participatory culture in the creation of academic knowledge}

\author{
Maria-Josep Picó; Emilio Sáez; Esteban Galán
}

Cómo citar este artículo:

Picó, Maria-Josep; Sáez, Emilio; Galán, Esteban (2019). “Investigación transmedia. Cultura participativa en la creación del conocimiento académico". El profesional de la información, v. 28, n. 4, e280414.

https://doi.org/10.3145/epi.2019.jul.14

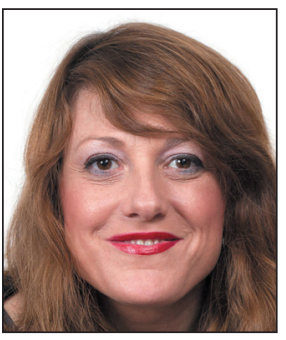

Maria-Josep Picó $\triangle$

https://orcid.org/0000-0002-7086-2269

Universitat Jaume I

Departamento de Ciencias de la

Comunicación

Av. Sos Baynat, $\mathrm{s} / \mathrm{n}$.

12071 Castellón de la Plana, España

mpico@uji.es

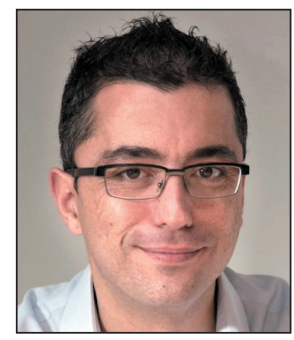

Esteban Galán

https://orcid.org/0000-0001-8718-0937

Universitat Jaume I

Departamento de Ciencias de la

Comunicación

Av. Sos Baynat, s/n.

12071 Castellón de la Plana, España

egalan@uji.es
Artículo recibido el 08-03-2019

Aceptación definitiva: 13-05-2019

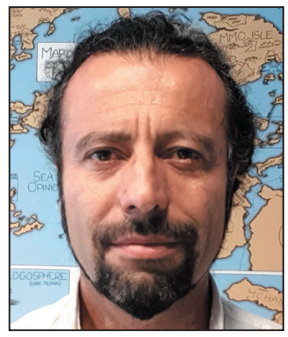

Emilio Sáez

https://orcid.org/0000-0001-7989-7698

Universitat Jaume I

Departamento de Ciencias de la

Comunicación

Av. Sos Baynat, s/n.

12071 Castellón de la Plana, España

soro@uji.es

\section{Resumen}

Se presenta un proyecto experimental de la Universitat Jaume I para utilizar narrativas transmedia con el objetivo de facilitar la participación en la comunicación de la ciencia. Conclusiones del proyecto Ágora Digital: narrativas transmedia y cultura participativa en la comunicación científica, respaldado por la Fecyt, en el que han participado miembros de diez grupos de investigación y el Centro Tecnológico Espaitec. Este estudio aborda las narrativas transmedia y la implementación de estrategias de ludificación o gamificación como una oportunidad para avanzar desde la comunicación hacia una investigación más responsable y alineada con las expectativas y valores de la ciudadanía. Los resultados del proyecto

\section{Financiación}

El presente trabajo ha sido realizado en el marco del proyecto de investigación Participación ciudadana y medios de comunicación públicos. Análisis de experiencias de co-creación audiovisual en España y en Europa (Parcicom) (código RTI2018-093649-B-I00), bajo la dirección de Javier Marzal Felici, financiado por la Convocatoria 2018 de Proyectos de I+D+I "Retos Investigación", del Programa Estatal de I+D+i orientada a los Retos de la Sociedad, para el período 2019-2021.

Así mismo, ha sido realizado en el marco del proyecto de investigación: Análisis de identidades discursivas en la era de la posverdad. Generación de contenidos audiovisuales para una Educomunicación crítica (Aidep) (código 18I390.01/1), bajo la dirección de Javier Marzal Felici, financiado por la Universitat Jaume I, a través de la convocatoria competitiva de proyectos de investigación para el período 2019-2021.

La iniciativa Ágora Digital ha contado con el apoyo de la Fundación Española para la Ciencia y la Tecnología (Fecyt-16-11023). 
muestran cómo acercar la comunidad investigadora a la comunicación institucional y han servido como experiencia piloto para el proyecto internacional Out of the box.

\title{
Palabras clave
}

Comunicación científica; Gamificación; Ludificación; Investigación; RRI; Participación; Innovación; Ciencia; Redes sociales; Transmedia.

\begin{abstract}
An experimental project of the University Jaume I, Castellón, Spain, to use transmedia narratives to facilitate the participation in the communication of science is presented. More precisely, it presents the conclusions of the project "Ágora Digital: narrativas transmedia y cultura participativa en la comunicación científica", backed by the Spanish Foundation for Science and Technology (Fecyt), in which members of ten research groups and the Technological Center Espaitec, have participated. This study approaches transmedia narratives and the implementation of gamification strategies as an opportunity to advance from the field of communication towards more responsible research, aligned with the expectations and values of the citizens. The results of the project has been useful to introduce the institutional communication channels to the research community of the University Jaume I and as a pilot of the international project Out of the box.
\end{abstract}

\section{Keywords}

Science communication; Research; RRI; Participation; Innovation; Science; Social Media; Transmedia.

\section{Introducción y revisión de la bibliografía}

El proyecto Ágora Digital impulsado desde el Servicio de Comunicación y Publicaciones y el Departamento de Ciencias de la Comunicación de la Universitat Jaume I ha tenido como objetivo implantar las narrativas transmedia (Jenkins; Ford; Green, 2015; Sánchez-Mesa et al., 2016; Scolari, 2018; Gosciola; Ribeiro, 2018; Pratten, 2018) y la ludificación o gamificación (Dicheva et al., 2015; Wiggins, 2016) en la comunicación científica y en la promoción de la investigación responsable. Gracias a las redes sociales, las formas de comunicar e interaccionar en la era digital afectan a los medios de comunicación convencionales y tienen importantes repercusiones narrativas y económicas. Además, en el ámbito científico están abriendo nuevas posibilidades para innovar en la difusión de la ciencia y promover el diálogo entre la comunidad académica y la sociedad e implantar en las universidades la cultura digital y el conocimiento abierto (García-Peñalvo; Ramírez-Montoya, 2015; Senabre; Ferran-Ferrer; Perelló, 2018; Chomón-Serna; Busto-Salinas, 2018). Sin embargo, estas posibilidades educativas y de servicio público no han sido exploradas suficientemente, siendo en gran medida las grandes productoras de contenido (Disney, Warner Bros o Universal) o las plaLas nuevas formas de comunicar, en especial las redes sociales, están abriendo nuevas posibilidades para innovar en la difusión de la ciencia y promover el diálogo entre la comunidad académica y la sociedad

taformas de vídeo a la carta (Netflix, Amazon, HBO o Movistar en España) quienes han capitalizado hasta el momento los proyectos de narrativas transmedia de mayor éxito con series y películas que invierten una parte importante de su presupuesto en la creación de un universo transmedia cuya finalidad es la promoción de la propia franquicia o del merchandising que el contenido lleva asociado (Galán, 2016). Es por esta razón por la que entendemos que es relevante llevar a cabo proyectos en los que se implementen narrativas transmedia al servicio de la innovación y de la creación y divulgación del conocimiento.

Para que esta estrategia comunicativa llegue de manera efectiva a la sociedad, se ha decidido incorporar junto con las narrativas transmedia, una experiencia de gamificación (Pérez-Manzano; Almela-Baeza, 2018). El objetivo de fusionar la lógica comunicativa transmedia con prácticas de gamificación es conseguir un salto cualitativo en los usos que los científicos realizan de los medios de comunicación (Schouten et al., 2019). La lógica de los juegos estimula la acción comunicativa de los involucrados para obtener resultados que conectan con sus intereses (Maltseva; Fieseler; Trittin-Ulbrich, 2019).

\subsection{Hacia una investigación e innovación responsable}

La investigación y la innovación se han convertido en piezas clave para el desarrollo social por su importancia como motor del progreso económico y social (Friesenhahan; Beaudry, 2014). Por ello, el debate sobre qué investigar, a qué áreas destinar más recursos y esfuerzos, emerge como un elemento capital de las democracias contemporáneas (García-Marzá; Fernández-Beltrán; Sanahuja, 2017). Todas estas cuestiones han suscitado en los últimos años la aparición de un nuevo concepto, el de la investigación e innovación responsables (más conocido como RRI, responsible research and innovation), que ha sido promovido por la Unión Europea, y que plantea la exigencia de que comunidad científica y sociedad trabajen juntas para que los procesos y resultados de la ciencia respondan a las expectativas y valores del conjunto de la ciudadanía y no sólo de los investigadores (García-Marzá, 2017). En este sentido, la RRI (European Commission, 2012) se puede ver como un proceso fruto de la conjunción entre la ética y la comunicación y es, por tanto, un concepto elaborado desde el conocimiento humanístico y que aporta valor al conjunto de la ciencia (Owen; Macnaghten; Stilgoe, 2012). 
Este modelo sitúa la comunicación pública de la ciencia en su centro haciendo imposible separar los procesos de trabajo y sus resultados de la actividad divulgadora. Para que esto sea posible es imprescindible la implementación de estrategias comunicativas que en el entorno actual necesariamente tienen que estar presentes en redes sociales con formatos audiovisuales porque son la fuente de información científica más consultada en Internet (Fecyt, 2017). El proyecto Ágora Digital es útil para que el reto lanzado por la Comisión Europea a través del concepto de investigación e innovación responsable (RRI) pueda contribuir a mejorar la visibilidad y la reputación de la comunidad investigadora (Rodríguez-Bravo; Nicholas, 2019).

\subsection{La narrativa transmedia como oportunidad para las instituciones académicas}

Transmedia es básicamente una manera de transmitir mensajes, es decir, de comunicar. Por tanto, el concepto de transmedia no atiende al mensaje sino al proceso de comunicar el mensaje (Jenkins et al., 2006, p. 46; Hernández-Ruiz, 2018; Sánchez-Mesa, 2019). Esto debe ser coherente con la cultura corporativa de la institución y por ello, para diseñar una estrategia transmedia de la comunicación científica, es necesario implantar de manera previa la cultura participativa orientada a la co-creación (Scolari, 2013; Deuze; Prenger, 2019). Así, se podrán compartir contenidos en los medios sociales en los que se muestren procesos internos del proyecto (primer borrador de un texto, el billete de tren para asistir a una conferencia o la imagen de un café en una reunión del grupo de investigación) que son los que tienen un mayor atractivo en los medios sociales (Cam-
El destinatario de la estrategia de comu- nicación transmedia deja de ser un indi- viduo y pasa a ser una comunidad pos-Freire et al., 2016; Serrano-Tellería, 2019). Las narrativas transmedia, aplicadas a la comunicación institucional, van más allá de una simple combinación de relatos y plataformas, definimos y entendemos la comunicación transmedia de una institución o proyecto como señalan Galán, Rodríguez-Serrano y Marzal-Felici:

"El plan o estrategia de comunicación integral que busca establecer una relación transparente y de confianza con su comunidad" (Galán; Rodríguez-Serrano; Marzal-Felici, 2018).

De esta manera se crean las condiciones para que pueda existir un diálogo entre la sociedad y los investigadores (Díaz-Noci; Tous-Rovirosa, 2012).

\section{3. ¿Es posible implementar estrategias de gamificación en una institución académica?}

Entendemos por gamificación el uso de mecánicas de juego en contextos que previamente no eran considerados lúdicos; un ejemplo lo podemos encontrar en su uso en la adopción de decisiones en nuestra vida cotidiana o en el ámbito laboral o familiar (McGonigal, 2011). Este tipo de estrategia lúdica, por su propia naturaleza, es transmedia dado que abarca diferentes tipos de comunicación (mediatizada e interpersonal) y plataformas (unidireccionales e interactivas) (Coombs, 2019). La gamificación, en definitiva, depende de la intención de quien diseña la experiencia y de las expectativas de quienes participan en ella y por ello es fundamental el sentido con que se cree dicha estrategia y los objetivos que persiga (Furini; Mirri; Montangero, 2019). No existen recetas mágicas para diseñar una experiencia gamificada, su éxito dependerá de que los intereses y motivaciones que persigan sus destinatarios sean coherentes con los objetivos de quien implementa dicha estrategia (Deterting, 2012). La pervivencia en el tiempo de esta estrategia depende de la capacidad que tenga su creador para añadir valor dentro de dicha experiencia (Kappen; Nacke, 2013), teniendo además en cuenta que el sentido de la gamificación no se justifica únicamente de manera individual: el concepto de comunidad es clave porque los individuos que componen una comunidad son más proclives a interactuar entre sí y con otros y por tanto, la experiencia de juego es más intensa y agradable (Lounis; Pramatari; Theotokis, 2014; Hamari; Koivisto, 2013). Para delimitar el concepto de gamificación y su alcance dentro de este proyecto

La apuesta por la gamificación dentro de este proyecto permite crear sinergias entre las plataformas de divulgación de contenidos y fortalecer el crecimiento de una comunidad alrededor de ellas es importante tener en cuenta que cualquier proyecto susceptible de ser gamificado debe tener sentido previamente a la implementación de las estrategias de juego (Deterting, 2011; Huotari; Hamari, 2012). La apuesta por la gamificación dentro de este proyecto supone una excelente oportunidad para crear sinergias entre plataformas y fortalecer el crecimiento de la comunidad.

Ágora Digital (figura 2) ha buscado una estrategia de gamificación que combine la comunicación interpersonal con los contenidos digitales para facilitar el trasvase de conocimiento. El proyecto propone un cambio radical en la cultura comunicativa del colectivo involucrado. Se plantea ampliar los canales de comunicación tradicionales de los investigadores (revistas, libros y congresos) para introducir medios sociales como Twitter y otros de carácter más especializado como Academia.edu o ResearchGate. Estos cambios siempre conllevan resistencias porque modifican parte del trabajo coti- 
diano del investigador y por ello se ha considerado fundamental la implementación de estrategias de gamificación que faciliten el proceso.

\section{Objetivos del proyecto Ágora Digital}

Este trabajo tiene como objeto fundamental analizar el impacto generado por el proyecto Ágora digital: narrativas transmedia y cultura participativa en la comunicación científica, desarrollado por la UCC+i de la Universitat Jaume I durante ocho meses (abril a diciembre de 2017), en el que han participado diez grupos y dos institutos de investigación de la Universitat Jaume I, además de Espaitec, el Parque Científico, Tecnológico y Empresarial de esta universidad y tres de sus empresas, 4TIC, Outcome's10 y Solucions Cuatroochenta. En concreto, han participado los grupos: Neurobiotecnología, Neuroanatomía, Unisexsida, Itaca, Great, Social Innova, Ética y Democracia, GROC, Semiconductores Avanzados (GAS) y Ecofisiología y Biotecnología.

También han participado miembros del Instituto Interuniversitario de Desarrollo Local (IIDL) de las Facultades de Ciencias Jurídicas y Económicas y de Ciencias Humanas y Sociales, y el Instituto de Materiales Avanzados (INAM) de la Escuela Superior de Tecnología y Ciencias Experimentales.

Se trata de una investigación fundamentalmente cualitativa cuyo objetivo es dotar de herramientas y hábitos de divulgación científica a los investigadores en el entorno digital y, a su vez, monitorizar el nivel de desempeño de los mismos a través del análisis de las métricas y de cuestionarios.

\section{Material y métodos}

Para lograr estos objetivos se ha optado por una metodología cualitativa (Corbetta, 2010, p. 41) a través de un planteamiento de investigación-acción complementada con la organización de grupos de discusión y la realización de entrevistas a los participantes. Es especialmente destacable la aportación cualitativa que ha supuesto para el proyecto la información extraída de los grupos de discusión. Aunque no se ha seguido de manera estricta una única metodología, sí que se han implementado en la organización de estos focus groups técnicas de dinamización, pensamiento creativo y co-creación (Harvey; Holmes, 2012) que permitieran sesiones semi-estructuradas buscando que el moderador de las sesiones estuviera al mismo nivel que los participantes y que a su vez entre los participantes, tampoco existieran jerarquías o diferencias marcadas por su nivel académico o por su condición de experto en la materia. El carácter semi-estructurado de estas sesiones se ha revelado decisivo para que la creatividad y la serendipia surgieran de manera natural en el desarrollo de las mismas y de esta manera pudieran surgir planteamientos originales que supusieron avances significativos para el proyecto. Estas técnicas se han complementado con el análisis cuantitativo de las métricas generadas por las publicaciones en Twitter y el blog colectivo.

\section{Investigación-acción}

Se ha adoptado una metodología de investigación-acción como estrategia participativa que permite al equipo de investigación intervenir en el objeto de estudio para mejorar sus prácticas en una espiral de ciclos de planificación, acción, observación y reflexión. La acción propuesta por el proyecto Ágora Digital tenía como objetivo la puesta en marcha de un proyecto transmedia y de gamificación para la divulgación científica.

\section{Análisis de las métricas}

En segundo lugar, se han aplicado técnicas de investigación cuantitativas a través del análisis de las métricas que proporcionan las plataformas de contenidos online y los medios sociales para estudiar el nivel de aceptación de las estrategias transmedia y de gamificación implementadas en el proyecto. En este texto se analizan las cifras de participación de 96 investigadores en el blog ÁgoraDigital y la actividad en la red social Twitter con las etiquetas \#ÁgoraDigital y \#CiènciaUJ durante el proyecto.

\section{Entrevistas a los científicos miembros del proyecto}

Se ha empleado una metodología cualitativa, mediante el diálogo con los científicos que han participado en Ágora Digital y la elaboración de unas entrevistas con el fin de obtener una radiografía previa y posterior al proyecto sobre la proximidad de la comunidad investigadora de la UJI a la información científica institucional.

Los objetivos de este primer análisis de Ágora Digital han consistido en responder a las siguientes preguntas de investigación:

- ¿Qué nivel de implicación y proyección ha logrado la implantación del modelo de difusión científica transmedia Ágora Digital?

- ¿Qué ventajas e inconvenientes presenta la estrategia transmedia y de gamificación de la comunicación científica?

\section{Resultados}

A continuación, se muestran los resultados obtenidos en la implantación de una cultura participativa en los grupos de investigación a través de las narrativas transmedia y la gamificación. Se considera que el nivel de incorporación de la comu- 
nidad investigadora a las redes sociales y los lenguajes digitales para difundir los conocimientos científicos ha sido moderado. Como fortalezas, conviene destacar la implicación de los investigadores, que se han convertido en usuarios habituales de las redes y han creado su comunidad, tanto local como internacional, transformándose además en un ejemplo para el resto de investigadores.

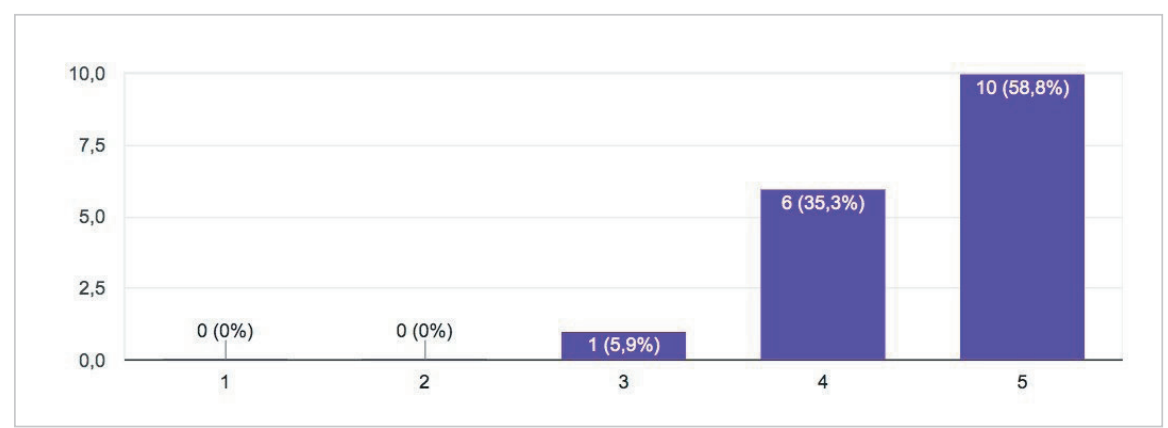

Gráfico 1. Resultados de la encuesta de satisfacción a los participantes $(4=$ satisfecho, $5=$ muy satisfecho).

Entre las debilidades cabe subrayar la dificultad para conseguir que las conversaciones digitales sobre ciencia de la comunidad investigadora de la Universitat Jaume I en Twitter amplíen su impacto a nuevos sectores de la sociedad. Otra cuestión a mejorar es la incorporación de criterios de gamificación en la comunicación científica transmedia que estén alineados con las prioridades de los investigadores.

Por otra parte, destaca el nivel elevado de satisfacción de los investigadores participantes en Ágora Digital (gráfico 1), mientras que esta iniciativa ha logrado un incremento del uso de redes sociales del 64,7\% (gráfico 2) en el colectivo de los investigadores implicados en el proyecto, según los resultados de la entrevista realizada a los participantes tras la conclusión del proyecto, en enero de 2018.

\subsection{Implementación de la estrategia transmedia y de gamificación}

La estrategia transmedia implementada ha tenido en cuenta la necesidad de llevar a cabo acciones online y presenciales.

Como acciones online se optó por el blog Ágora Digital y por Twitter con los mensajes publicados con la etiqueta \#ÁgoraDigital. Con respecto a la elección de las redes sociales hubo debate porque Facebook era la red social más utili-

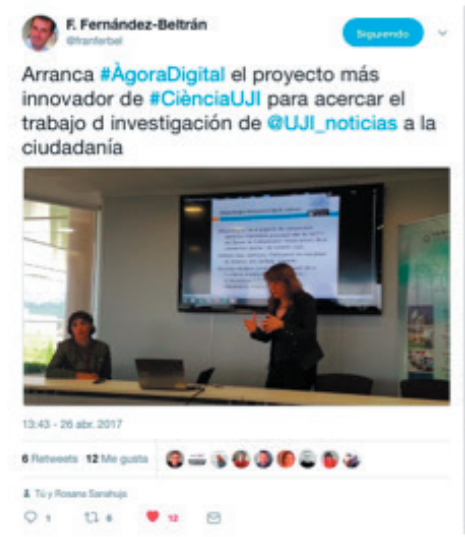

\section{Esther Ramo}

\#ÄgoraDigital, creando comunidad en la UJI para \#comunicar la ciencia de una forma diferente. Gasociacionruvid, te apuntas? \#CiènciaUul

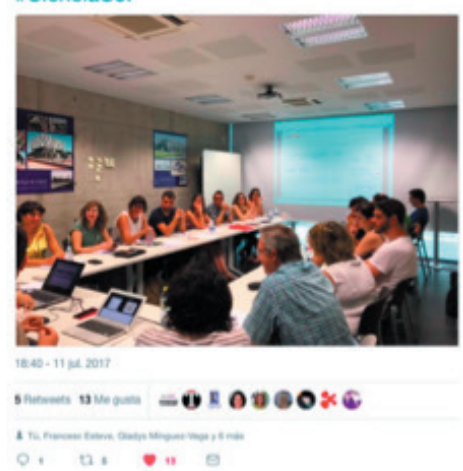

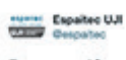

Presentación proyecto "AgoraDigital, comunicación cientifica para explicar a la ciudadanía la investigación desarrollada en EUVI_noticlas

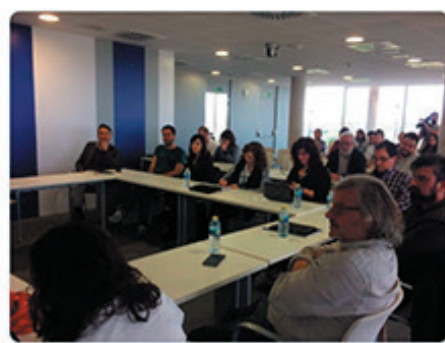

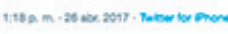

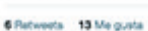

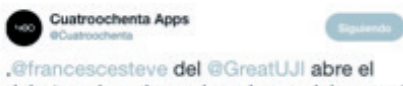
debate sobre el uso de redes sociales en el ámbito cientifico en ili \#ClènciaU.Ji

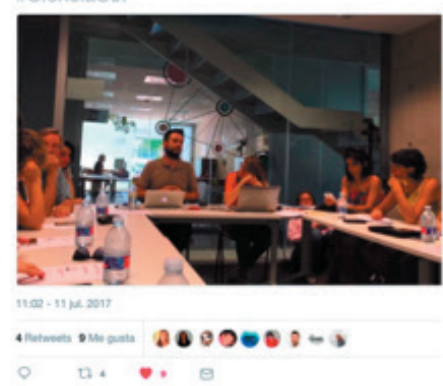

Maria Josep Picó Emariajpico - 28 jun. 2017

Tres empreses Eespaitec a ithoorabigital ICitncialu. biogsuli. es/agoradigta/2....

Donem al benvinguda a CCuatroochenta eOutcomesi $10 \mathrm{i}$ Courtic

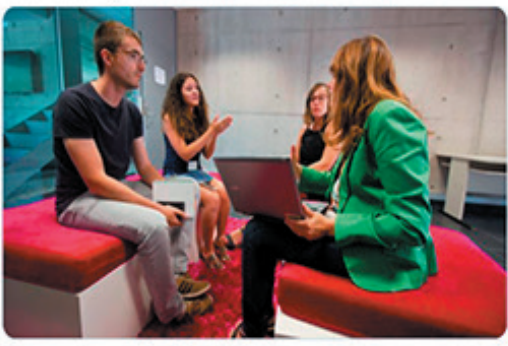

UnWersitat Jaume ly 9 más

$\begin{array}{llll}0 & 27 & 0 & 12\end{array}$

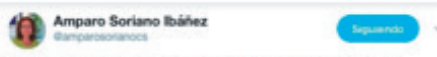

Encantada de compartir lo aprendido en \#DSMValencia con los compañeros de QUJI_noticias en \#CiènciaUJII \#ÄgoraDigital

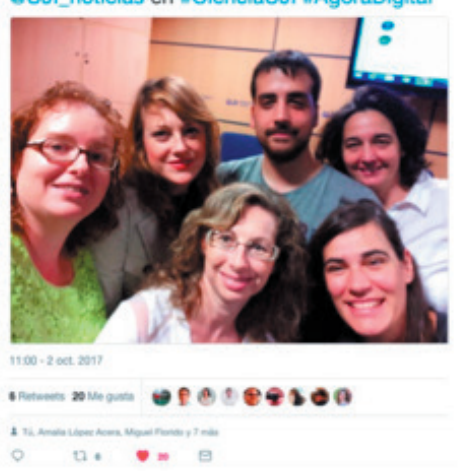

Figura 1. Tweets de la organización de Grupos de interés y sesiones formativas del proyecto Ágora Digital 
zada por los investigadores del proyecto e Instagram era la red social con más incremento de seguidores. Sin embargo, Twitter ofrece la ventaja de ser una plataforma donde los usuarios publican mensajes que no tienen normalmente relación con su vida privada y esto se entendió que era importante para conseguir una mejor tasa de participación de los investigadores.

Dentro de las acciones presenciales se optó por organizar grupos de discusión con el objetivo de decidir las acciones a realizar y acciones formativas dirigidas a solucionar las carencias detectadas. Se puede acceder al cuestionario y al contenido completo de los grupos de discusión en el documento:

https://bit.ly/309K2rj

El primer grupo de discusión, celebrado el 26 de abril de 2017, sirvió para decidir las plataformas a utilizar (blog y Twitter) y las acciones formativas (redes sociales y escritura creativa). En esa primera reunión se discutió sobre el concepto de investigación e innovación responsable (RRI) y su relación con la comunicación transmedia. Se constató además el desconocimiento que los investigadores tienen de lo que se investiga en su propia universidad. Se detectó también la necesidad de organizar actividades formativas sobre aspectos como la escritura para redes sociales y el manejo de la identidad digital. Para solucionar esas dos carencias detectadas, se decidió organizar dos sesiones formativas presenciales para los miembros del proyecto con el fin de reforzar el establecimiento de lazos entre los investigadores.

Tras estas sesiones tuvo lugar un segundo grupo de discusión, el 11 de julio de 2017, en el que se diseñó la estrategia de gamificación a plantear para el proyecto. Se buscaba un elemento con el que se pudieran identificar los investigadores de los diferentes campos envueltos en el proyecto. Desde este objeto de atención se planteaba crear un flujo de comunicación que pudiera ser compartido. Se trataba de crear contenidos que pudiesen ser objeto de comunicación y que además fuesen el objetivo de una dinámica de gamificación que resultase estimulante para los participantes. Se decidió que un objeto relevante en pleno verano, podría ser relatar los aspectos de común interés en un paraje natural cercano, en este caso la Sierra de Irta, anexa al mar entre los municipios de Alcoceber y Peñíscola en la provincia de Castellón (España). Se optó por una mecánica consistente en desplazarse al lugar y ser capaces de suscitar la mayor atención posible creando y distribuyendo contenido sobre este paraje en relación con el área de investigación propia. Este juego suponía el reto de:

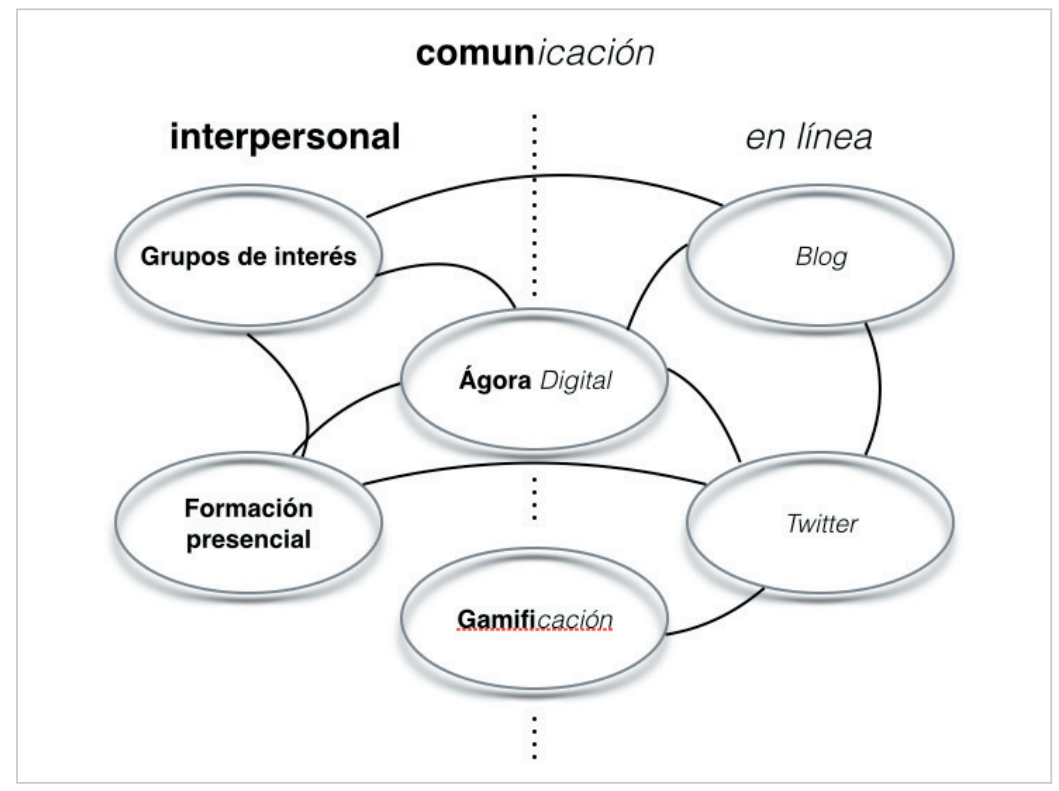

Figura 2. Esquema de las acciones desarrolladas dentro del proyecto Ágora Digital y de la conexión existente entre ellas.

- plantear la necesidad de concretar sus saberes en relación con un objetivo común (creación de comunidad);

- describir qué elementos eran importantes en relación con su área para comprender dicho entorno (creación de relevancia);

- su capacidad para establecer una comunicación con la sociedad de esos conocimientos (interactividad);

- conocer sus resultados en el reto, los propios y en comparación con los demás participantes (progreso).

De esta manera, se completaba el mapa de acciones planteadas en el proyecto que se muestran en la figura 2.

Una debilidad detectada a posteriori en lo que respecta a la estrategia de gamificación, es que solamente se ha conectado la gamificación con una plataforma: Twitter. Esta circunstancia probablemente haya limitado el alcance de dicha iniciativa. Queda pendiente incorporar en próximos proyectos la gamificación como eje vertebrador también en las iniciativas de comunicación interpersonal.

\subsection{Análisis de las métricas de la actividad online}

La iniciativa Ágora Digital ha logrado la implicación formal de miembros de diez grupos de investigación y dos institutos de investigación y el Parque Científico, Tecnológico y Empresarial, Espaitec, y tres de las empresas radicadas en este espacio. Además, durante su desarrollo también ha facilitado la implicación individual de investigadores de otros grupos y departamentos de la Universidad. A su vez, se ha consolidado como un espacio de interacción, conocimiento y colaboración interdisciplinar de profesionales de las diversas áreas de conocimiento, como revela la creación del Seminario Permanente de Investigadores en Cambio Climático y también como un área de formación para la comunicación científica y la conformación de la identidad digital de la comunidad investigadora. 
El proyecto de comunicación científica transmedia se ha sustentado en dos medios principales:

- participación en un blog colectivo denominado Ágora Digital; y

- uso de Twitter, empleando las etiquetas \#ÁgoraDigital y \#CienciaUJI.

Se han analizado los tweets que utilizaron las etiquetas del proyecto \#ÁgoraDigital, en comparación también con otras etiquetas, como la genérica \#CienciaUJI. La aplicación utilizada ha sido Follow the hashtag:

http://www.followthehashtag.com

La figura 3 muestra la evolución y alcance de \#ÁgoraDigital

Los datos más destacados de la actividad en Twitter con la etiqueta \#ÁgoraDigital durante un período de 282 días muestran que los mensajes con este marcador lograron una audiencia total de 126.507, mientras que las impresiones totales fueron de 493.446. En total, se contabilizaron 145 tweets de 52 perfiles que obtuvieron 97 retweets y que compartieron 34 imágenes.

Sobre el incremento del uso de las redes sociales por parte de la comunidad investigadora de la $U \mathrm{Jl}$, el $41,2 \%$, reconoce que el proyecto ha motivado su uso de Twitter para publicar contenidos relacionados con su investigación, mientras que

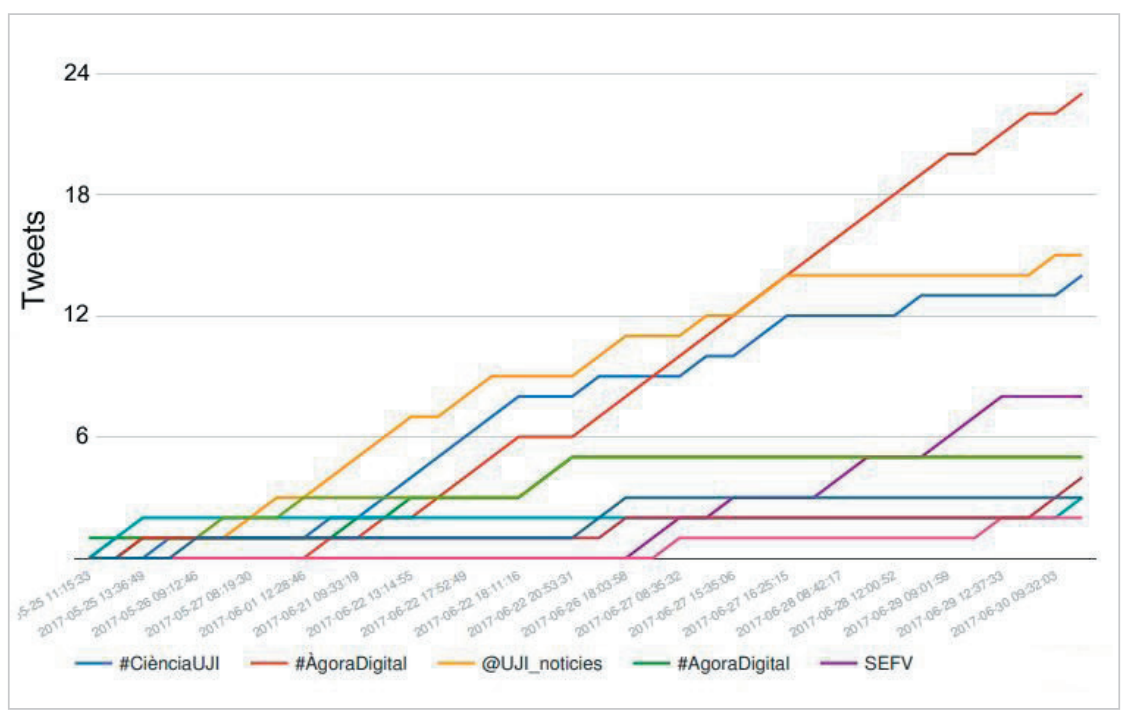

Gráfico 2. Información agregada sobre las citaciones de marcadores y perfil de la Universidad el 23,5\% asegura que Ágora Digital ha hecho posible su primera aproximación a las redes sociales. En consecuencia se ha incrementado un 64,7\% la utilización de Twitter para la comunicación científica entre los investigadores que participaron.

El blog Ágora Digital logró aglutinar un total de 60 textos de diversos investigadores, es decir, una media de 7,5 entradas mensuales, una cantidad que se considera relevante y que ha servido de complemento y refuerzo a la actividad de la comunicación institucional de los resultados de investigación de la Universidad.

http://blogs.uji.es/agoradigital

\subsection{Entrevistas semiestructuradas con los investigadores}

Las entrevistas semiestructuradas en profundidad realizadas a 17 científicos miembros de los grupos de investigación de la UJl que han participado en la iniciativa Ágora Digital han permitido conocer una percepción cualificada de las fortalezas y debilidades de la implantación de un modelo de comunicación científica transmedia en una institución de educación superior como la universidad pública de Castellón. Las líneas de diálogo fueron siete:

1) frecuencia en el uso de los medios digitales informativos de la $\mathrm{UJ}$;

2) calificación global Ágora Digital;

3) experiencias personales de la participación;

4) cambios en el uso de las redes sociales;

5) valoración de Twitter como red para compartir contenidos científicos;

6) Importancia de establecer un diálogo interactivo ciencia-sociedad;

7) Influencia de las expectativas de la ciudadanía en su investigación científica.

Entre las fortalezas y ventajas destacamos los cinco argumentos más relevantes, de mayor a menor importancia, según los investigadores:

- poder llegar a los ciudadanos de manera inmediata;

- un medio más próximo, rápido, interactivo, accesible;

- medio que favorece un tono más informal para comunicar la ciencia, con más proximidad a los ciudadanos;

- posibilidad de obtener feedback desde diferentes sectores;

- facilitar la transferencia de los resultados a la sociedad.

A continuación, las desventajas o debilidades, también por orden de prioridad en las respuestas:

- la rapidez de las redes no favorece la reflexión;

- falta de habilidades digitales y tiempo;

- excesiva brevedad, frente a la complejidad científica;

- peligro de crear falsas expectativas y demasiado 'ruido';

- riesgo de exceso de protagonismo de algunos investigadores. 


\section{Conclusiones}

La investigación ha conseguido responder de manera satisfactoria a las dos preguntas inicialmente planteadas sobre las ventajas e inconvenientes de la implantación de una cultura participativa en la creación y difusión del conocimiento a través de estrategias transmedia y de gamificación. En este sentido, los resultados de este estudio constituyen un punto de partida para implantar un modelo de comunicación científica transmedia en la universidad pública y entendemos que los procesos detallados pueden ser replicados en otras instituciones.

Las conclusiones del proyecto muestran, en primer lugar, una aproximación moderada de la comunidad investigadora de la Universidad a los canales de comunicación institucionales al tiempo que revelan un destacado potencial de innovación en la difusión científica liderada por los propios investigadores donde se promueve una comunicación científica más próxima a los ciudadanos. Este estudio también revela que mientras que la comunidad científica aprueba el diálogo entre ciencia y sociedad, los investigadores muestran reservas a que las expectativas de los ciudadanos puedan influir en la determinación de las áreas de investigación científica que se deben potenciar, en línea con la Responsible Research and Innovation (European Commission, 2014).

Respecto al planteamiento de la estrategia transmedia, los investigadores han constatado la dificultad de divulgar a través de varias plataformas (en este caso blog y Twitter) sin repetir el contenido. Se ha identificado como un aspecto clave la dinamización de los canales de comunicación interna, es decir: para empezar a comunicar hay que fortalecer la comunicación interna para generar una comunidad alrededor del investigador. Esto es todavía más importante en el escenario transmedia en el que la frontera entre comunicación interna y externa aparece cada vez más borrosa. El científico debe dialogar con los individuos en quienes impacta su investigación aunque eso termine implicando una cierta pérdida de poder en la elección de sus líneas de investigación (para la que los científicos no están todavía preparados).

En lo referente a la iniciativa de gamificación podemos valorarla de forma positiva en la medida en que en el grupo de discusión se trató una metodología novedosa en cuanto a su interés como forma de dinamizar todo tipo de actividades en grupo y en concreto como vía para una mejor integración de las prácticas de comunicación en red. La experiencia planteada tuvo un seguimiento discreto con cuatro iniciativas de creación de contenido desde diferentes perspectivas del conocimiento, pero suficiente para mostrar a los participantes en el proyecto la sencillez y eficacia del planteamiento. Los hallazgos obtenidos en el proyecto Ágora Digital que se basan fundamentalmente en la instalación de una cultura participativa en la que se fomente la investigación interdisciplinar dentro de las organizaciones, han servido para poner en marcha juntamente con la Glasgow Caledonian University el proyecto Out of the box que en enero de 2019 ha celebrado con éxito su primera edición con más de 20 investigadores de cuatro instituciones pertenecientes a 3 países y que en los próximos meses celebrará un segundo encuentro en Castellón. Out of the box ha supuesto una oportunidad para aplicar a nivel internacional los resultados del proyecto Ágora Digital que hemos presentado en este trabajo.

https://comtransmedia.com/out-of-the-box

\section{Limitaciones}

La principal limitación del presente trabajo se debe a que sólo se ha llevado a cabo el experimento en una única institución. Sin embargo, la cantidad de investigadores involucrados en el proyecto, su carácter interdisciplinar, junto con el período de aplicación del mismo (8 meses), ofrecen un campo de experimentación interesante que puede servir para ser replicado en otras instituciones.

\section{Referencias}

Campos-Freire, Francisco; Rúas-Araújo, José; López-García, Xosé; Martínez-Fernández, Valentín-Alejandro (2016). "Impacto de las redes sociales en el periodismo". El profesional de la información, v. 25, n. 3, pp. 449-457. https://doi.org/10.3145/epi.2016.may.15

Chomón-Serna, José María; Busto-Salinas, Lorena (2018). “Ciencia y transmedia: binomio para la divulgación científica. El caso de Atapuerca". El profesional de la información, v. 27, n. 4, pp. 938-946. https://doi.org/10.3145/epi.2018.jul.22

Coombs, Timothy (2019). "Transmedia storytelling: A potentially vital resource for CSR communication". Corporate communications: An international journal, v. 24, n. 2, pp. 351-367.

https://doi.org/10.1108/CCIJ-11-2017-0114 
Corbetta, Piergiorgio (2010). Metodología y técnicas de investigación social. Madrid: McGrawHill. ISBN: 9788448156107 https://diversidadlocal.files.wordpress.com/2012/09/metodologc3ada-y-tc3a9cnicas-de-investigacic3b3n-socialpiergiorgio-corbetta.pdf

Deterting, Sebastian (2012). “Gamification: Designing for motivation”. Interactions, v. 19, n. 4, pp. 14-17. https://doi.org/10.1145/2212877.2212883

Deuze, Marc; Prenger, Mirjam (ed.) (2019). Making media. Production, practices, and professions. Amsterdam: Amsterdam University Press. ISBN: 9789048550708

Díaz-Noci, Javier; Tous-Rovirosa, Anna (2012) "La audiencia como autor: narrativas transmedia y propiedad intelectual del público. Algunas reflexiones jurídicas”. El profesional de la información, v. 21, n. 5, pp. 458-467.

https://doi.org/10.3145/epi.2012.sep.03

Dicheva, Darina; Dichev, Christo; Agre, Gennady; Angelova, Galia (2015). “Gamification in education: A systematic mapping study". Journal of educational technology \& society, v. 18, n. 3, pp. 75-88.

https://www.jstor.org/stable/jeductechsoci.18.3.75

European Commission (2012). Responsible research and innovation. Europe's ability to respond to societal challenges. Bruselas: Publicaciones Oficiales UE.

https://ec.europa.eu/research/swafs/pdf/pub_rri/KIO214595ENC.pdf

Fecyt (2017). VIII Encuesta de percepción social de la ciencia y la tecnología. Madrid: Fundación Española para la Ciencia y la Tecnología.

http://www.ciencia.gob.es/stfIs/MICINN/Cultura/FICHEROS/2017/Dossier_PSC_2017.pdf

Friesenhahn, Irene; Beaudry, Catherine (2014). The global state of young scientists. Project report and recommendations. Berlin: Akademie Verlag. ISBN: 9783939818441

http://www.interacademies.org/24897/The-Global-State-of-Young-Scientists-Project-Report-and-Recommendations

Furini, Marco; Mirri, Silvia; Montangero, Manuela (2019). "Gamification and accessibility". In: $16^{\text {th }}$ IEEE Annual consumer communications \& networking conf (CCNC), pp. 1-5. IEEE.

Galán, Esteban (2016). "Relato transmedia vinculado a marcas: el personaje de Benito como instrumento de branded content". El profesional de la información, v. 25, n. 6, pp. 915-922.

https://doi.org/10.3145/epi.2016.nov.08

Galán, Esteban; Rodríguez-Serrano, Aarón; Marzal-Felici, Javier (2018). Contenidos transmedia para la radiotelevisión de proximidad. Pamplona: Eunsa. ISBN: 9788431332877

García-Marzá, Domingo (2017). "From ethical code to ethical auditing: An ethical infrastructure for social responsibility communication". El profesional de la información, v. 26, n. 2, pp. 268-276.

https://doi.org/10.3145/epi.2017.mar.13

García-Marzá, Domingo; Fernández-Beltrán, Francisco; Sanahuja, Rosana (2017). Ética y comunicación en la gestión de la investigación e innovación responsables (RRI): el papel de las Unidades de Cultura Científica y de la Innovación (UCC+i). Publicacions de la Universitat Jaume I, Castelló de la Plana. ISBN: 9788416546176

https://doi.org/10.6035/Humanitats.2017.52

García-Peñalvo, Francisco-José; Ramírez-Montoya, María-Soledad (2015). “Educational innovation with a multicultural perspective". Journal of cases on information technology, v. 17, n. 1, pp. iv-v.

https://www.semanticscholar.org/paper/Educational-Innovation-with-a-Multicultural-Garcia-Penalvo-Montoya/278bc 9f21589b29ae56943adce67211226107610

Gosciola, Vicente; Ribeiro, Matheus T. V. (2018). “Midiatização pelos jovens na expansão narrativa do universo cinemático Marvel". Chasqui. Revista latinoamericana de comunicación, n. 137, pp. 111-128.

https://www.redalyc.org/jatsRepo/160/16057171014/index.html

https://doi.org/10.16921/chasqui.v0i137.3456

Hamari, Juho; Koivisto, Jonna (2013). "Social motivations to use gamification: an empyrical study of gamifying exercise". In: ECIS 2013, Completed Research, 105.

http://aisel.aisnet.org/ecis2013_cr/105

Harvey, Nichole; Holmes, Colin A. (2012). "Nominal group technique: An effective method for obtaining group consensus". International journal of nursing practice, v. 18, n. 2, pp. 188-194.

https://doi.org/10.1111/j.1440-172X.2012.02017.x

Hernández-Ruiz, Javier (2018). “Diseñando mundos transmedia: el caso Plot 28 (2013)”. Revista latina de comunicación social, n. 74, pp. 12-32.

http://www.revistalatinacs.org/074paper/1319/02es.html_ 
Huotari, Kai; Hamari, Juho (2012). "Defining gamification: A service marketing perspective". In: Proceedings of the $16^{\text {th }}$ Intl academic mindtrek conf, Tampere, Finland, Oct. 3-5, pp. 17-22.

https://doi.org/10.1145/2393132.2393137

Jenkins, Henry; Clinton, Katie; Puroshotma, Ravi; Robinson, Alice J.; Weigel, Margaret (2006). Confronting the challenges of participatory culture: Media education for the $21^{\text {st }}$ Century. Chicago, Illinois: The MacArthur Foundation. ISBN: 9780262258296

https://www.macfound.org/media/article_pdfs/JENKINS_WHITE_PAPER.PDF

Jenkins, Henry; Ford, Sam; Green, Joshua (2015). Cultura transmedia. La creación de contenido y valor en una cultura en red. Barcelona: Gedisa. ISBN: 9788497848459

Kappen, Dennis L.; Nacke, Lennart E. (2013). "The kaleidoscope of effective gamification: deconstructing gamification in business applications". In: Proceedings of the First intl conf on gameful design, research, and applications, pp. 119-122. ACM. ISBN: 9781450328159

https://doi.org/10.1145/2583008.2583029

Lounis, Stavros; Pramatari, Katerina; Theotokis, Aristeidis (2014). "Gamification is all about fun: The role of incentive type and community collaboration". In: Procs of the European conf on information systems (ECIS). Tel Aviv, Israel, June 9-11. http://aisel.aisnet.org/ecis2014/proceedings/track12/13

Maltseva, Kateryna; Fieseler, Christian; Trittin-Ulbrich, Hanna (2019). "The challenges of gamifying CSR communication". Corporate communications: An international journal, v. 24, n. 1, pp. 44-62.

https://doi.org/10.1108/CCIJ-09-2018-0092

McGonigal, Jane (2011). Reality is broken: Why games make us better and how they can change the world. Penguin, London. ISBN: 9781611064278

Owen, Richard; Macnaghten, Phil; Stilgoe, Jack (2012). "Responsible research and innovation: From science in society to science for society, with society". Science and public policy, v. 39, pp. 751-760.

https://doi.org/10.1093/scipol/scs093

Pérez-Manzano, Antonio; Almela-Baeza, Javier (2018). “Gamificación transmedia para la divulgación científica y el fomento de vocaciones procientíficas en adolescentes". Comunicar, v. 26, n. 55, pp 93-103.

https://doi.org/10.3916/C55-2018-09

Pratten, Robert (2018). "Elastic worlds". Icono 14, v. 17, n. 1, pp. 5-14.

https://doi.org/10.7195/ri14.v17i1.1302

Rodríguez-Bravo, Blanca; Nicholas, David (2019). “Reputación y comunicación científica: investigadores españoles en el inicio de su carrera". El profesional de la información, v. 28, n. 2, e280203.

https://doi.org/10.3145/epi.2019.mar.03

Sánchez-Mesa, Domingo (2019). Narrativas transmediales. La metamorfosis del relato en los nuevos medios digitales. Barcelona: Gedisa. ISBN: 9788417690632

Sánchez-Mesa, Domingo; Aarseth, Espen; Pratten, Robert; Scolari, Carlos A. (2016). "Transmedia (storytelling?): A polyphonic critical review". Artnodes, n. 18.

https://doi.org/10.7238/a.v0i18.3064

Scolari, Carlos A. (2013). Narrativas transmedia. Cuando todos los medios cuentan. Barcelona: Deusto. ISBN: 8423413365

Scolari, Carlos A. (2018). Alfabetismo transmedia en la nueva ecología de los medios. Libro blanco. Transliteracy. H2020 Research and Innovation Actions.

https://transmedialiteracy.org

http://transmedialiteracy.upf.edu/sites/default/files/files/TL_whit_es.pdf

Schouten, Ben; Van-der-Spek, Erik; Harmsen, Daniël; Bartholomeus, Ellis (2019). "The playful scientist: Stimulating playful communities for science practice". In: Glas, René; Lammes, Sybile; De-Lange, Michiel; Raessens, Joost; De-Vries. The playful citizen. Civic engagement in a mediatized culture. Amsterdam: Amsterdam University Press, pp. 235-254. ISBN: 9789462984523

Senabre, Enric; Ferran-Ferrer, Núria; Perelló, Josep (2018). “Diseño participativo de experimentos de ciencia ciudadana". Comunicar, v. 26, n. 54, pp. 29-38.

https://doi.org/10.3916/C54-2018-03

Serrano-Tellería, Ana (ed.) (2019). Between the public and private in mobile communication. Routledge. ISBN: 9781315399300

Wiggins, Bradley E. (2016). "An overview and study on the use of games, simulations, and gamification in higher education". International journal of game-based learning, v. 6, n. 1, pp. 1-29.

https://doi.org/10.4018/IJGBL.2016010102 